\title{
A NUMERICAL METHOD FOR SEMILINEAR SINGULAR PARABOLIC QUENCHING PROBLEMS*
}

\author{
BY \\ C. Y. CHAN ${ }^{1}$ AND C. S. CHEN \\ University of Southwestern Louisiana, Lafayette, Louisiana
}

\begin{abstract}
For the problem given by $u_{x x}+b u_{x} / x-u_{t}=-(1-u)^{-1}$ for $0<x<a$, $0<t<T_{a} \leq \infty, u(x, 0)=0=u(0, t)=u(a, t)$, where $b$ is a constant less than one, a lower bound of $u$ is used to estimate the critical length $a$ beyond which quenching occurs, and an upper bound for the time when quenching happens. An upper bound of $u$, given by the minimal solution of its steady state, is constructed by using a modified Picard method with the construction of the appropriate Green's function. To determine the critical length numerically, it is shown that for a given length $a$, all iterates attain their maximum values at the same $x$-coordinate; the largest interval for existence of the minimal solution corresponds to the critical length for the parabolic problem. As illustrations of the numerical method, the critical lengths corresponding to four given values of $b$ are computed.
\end{abstract}

1. Introduction. Let $T_{a}$ be the largest value such that a solution $u<1$ exists for the following semilinear parabolic initial boundary-value problem:

$$
\begin{gathered}
u_{x x}-u_{t}=-(1-u)^{-1}, \quad 0<x<a, \quad 0<t<T_{a} \leq \infty, \\
u(x, 0)=0 \text { for } 0 \leq x \leq a, \quad u(0, t)=0=u(a, t) \text { for } 0<t<T_{a} .
\end{gathered}
$$

If $T_{a}=\infty$, then $u(<1)$ exists globally. If $T_{a}<\infty$, then

$$
\lim _{t \rightarrow T_{a}^{-}} u\left(\frac{a}{2}, t\right)=1^{-} \text {, }
$$

where

$$
u(a / 2, t)=\max \{u(x, t): 0 \leq x \leq a\} .
$$

In the latter case, $u$ is said to quench at $T_{a}$ (cf. Acker and Walter [2]), and $T_{a}$ is called the quenching time. Equation (1.1) arises in the study of a polarization phenomenon in ionic conductors.

For practical purposes, the problem of quenching is to be able to find the critical length $a^{*}$ numerically such that $T_{a^{*}}=\infty$ and $T_{a}<\infty$ for $a>a^{*}$. Kawarada [17] showed that quenching occurs when $a>2^{3 / 2}$. Walter [27] showed that $1.5303 \leq a^{*} \leq$

*Received September 9, 1987.

${ }^{1}$ The work of this author was partially supported by the Board of Regents of the State of Louisiana under Grant LEQSF (86-89)-RD-A-11. 
$\pi / 2$. Acker and Walter [1] improved the above results by showing that $a^{*}=1.5303$ (to 5 significant figures). Naturally, quenching is due to the singularity on the righthand side of (1.1) when $u=1^{-}$.

Acker and Walter [1,2] studied the phenomena of quenching for the equations,

$$
\begin{aligned}
& u_{x x}-u_{t}=-f(u), \quad 0<x<a, \quad 0<t<T_{a}, \\
& u_{x x}-u_{t}=-g\left(u, u_{x}\right), \quad 0<x<a, \quad 0<t<T_{a},
\end{aligned}
$$

subject, respectively, to (1.2); for each problem, they showed that there is a unique critical length $a^{*}$. Results on the behavior of (1.3) subject to (1.2) with $a=a^{*}$ were given by Levine and Montgomery [22].

The case when the solution is driven by the boundary condition with the forcing term of the differential equation being identically zero was studied by Levine [19]. Higher space dimensional problems were investigated by Walter [27], Acker and Walter [1, 2], and Levine and Lieberman [21]. Related phenomena for hyperbolic equations were given by Chang and Levine [12], and Levine [19]. More recently, a survey was also given by Levine [20].

Here, we study the quenching phenomenon for the semilinear singular parabolic equation,

$$
L u=-(1-u)^{-1}, \quad 0<x<a, \quad 0<t<T_{a} \leq \infty,
$$

subject to $(1.2)$ with $b<1$, where

$$
L=\frac{\partial^{2}}{\partial x^{2}}+\frac{b}{x} \frac{\partial}{\partial x}-\frac{\partial}{\partial t} .
$$

When $b=0$, this reduces to the problem studied by Kawarada and Walter. The singular term $b u_{x} / x$ destroys the symmetry of the solution $u$ about the line $x=a / 2$, and shifts the points where $u$ attains its maxima with respect to $x$ from the line $x=$ $a / 2$. Thus, it makes the problem more difficult both theoretically and numerically.

If $b=0, L$ is the usual heat operator. When $b$ is a noninteger, it may be regarded as a first step in the approach to a theory of generalized axially symmetric heat potentials (cf. Alexiades [4], and Arena [7]). For $0 \leq b \leq 2$, it can be used to describe conduction of heat in a body with $b$ being a geometric parameter related to the shape of the body; for example, $b=0.5$ represents heat transfer into one face of a flat cylinder with a small ratio of depth to diameter (cf. Solomon [26]).

With $U(z, t)=u(x, t)$, where $z=2^{-1 / 2} x, L u$ is transformed into

$$
\frac{1}{2} U_{z z}+\frac{b}{2 z} U_{z}-U_{t}
$$

For $b>-1$, this corresponds to a stochastic process which is the limit of a sequence of random walks (cf. Lamperti [18]).

Let $W(Z, t)=u(x, t)$, where $Z=x^{2} / 4$. Then, $L u$ is transformed into

$$
Z W_{z z}+\frac{b+1}{2} W_{Z}-W_{t}
$$

This is a degenerate elliptic-parabolic expression occurring in the theory of probability (cf. Brezis, Rosenkrantz, and Singer with an appendix by Lax [9]). 
Cauchy problems for $L$ were investigated in many directions (cf. Arena [7], Bragg [8], Brezis, Rosenkrantz, and Singer with an appendix by Lax [9], Cholewinski and Haimo [13], Colton [14], and Haimo [15]). Nonhomogeneous initial boundary-value problems of the first, second, and third types for $L$ were studied by Alexiades [ 3 , 4] while Alexiades and Chan [5] considered a nonhomogeneous differential equation for $L$ subject to a nonlinear boundary condition. In these latter three papers, it was shown that for the problems to be well posed, no boundary condition at the singular axis $x=0$ can be assigned for $b \geq 1$, but for $b<1$, the first boundary condition is assigned at $x=0$. In particular, it follows from section 10 of Alexiades [4] that a classical solution $u$, in the case $b \geq 1$, has the property that $x^{b} u_{x}$ tends to zero as $x$ tends to zero; from section 11 there, we have existence of a unique classical solution for the first initial boundary-value problem: $L u=Q$, subject to (1.2), where $b<1$, and $Q(x, t)$ is a given function. By assuming that the problem (1.4) and (1.2) has a solution before its quenching time, our main purpose here is to give a method to determine the critical length $a^{*}$ numerically. Our study here may be said to be illustrative; we consider the nonlinear term $-(1-u)^{-1}$ only.

In Sec. 2, we use the method of Acker and Walter [1,2] to establish that there exists a unique critical length $a^{*}$. We then use a Sturm-Liouville problem to find a lower bound for the problem (1.4) and (1.2), and to obtain an estimate for $a^{*}$ as well as an upper bound for the time when quenching occurs. These estimates generalize the result of Walter. In Sec. 3, we use a modified Picard method (cf. Chan and Hon [11]) to construct a strictly monotone increasing sequence, converging uniformly to the minimal solution of the nonlinear singular two-point boundary value problem,

$$
\frac{d^{2} v}{d x^{2}}+\frac{b}{x} \frac{d v}{d x}=-(1-v)^{-1} \quad \text { for } 0<x<a, \quad v(0)=0=v(a) .
$$

We achieve this by constructing Green's function for the iterates through Bessel functions of the first kind and the second kind. This minimal solution provides an upper bound for the solution of the problem (1.4) and (1.2). To determine $a^{*}$ numerically, we show that for a given length $a$, all iterates attain their maximum values at the same $x$-coordinate $h$; furthermore, the behavior of the difference between two successive iterates at $h$ can be used to cut down the computer time for determining $a^{*}$. In Sec. 4 , we apply the numerical method, developed in Secs. 2 and 3, by using subroutines from the IMSL Library to compute the critical lengths corresponding to different values of $b$. In particular for $b=0$, we obtain the result of Acker and Walter [1]. Two graphs are also given to illustrate other results. Our numerical method is very stable since it involves mainly Newton's method of finding a root, and numerical integrations.

2. Critical lengths. By modifying the proofs of the corresponding theorems of Acker and Walter $[1,2]$ for the special case $b=0$, the following theorem can be established.

THEOREM 1. Let $u(x, t ; a)$ be a solution of the problem (1.4) and (1.2).

(a) There exists a curve $M(t)$ such that for each $t \in\left[0, T_{a}\right), u$ is monotone nondecreasing in $x$ on $[0, M(t)]$ and monotone nonincreasing in $x$ on $[M(t), a]$. 
(b) If $T_{a}=\infty$, and $u(x, t ; a) \leq A<1$ for some positive constant $A$, then

$$
v(x ; a)=\lim _{t \rightarrow \infty} u(x, t ; a)
$$

exists uniformly on $[0, a]$, where $v(<1)$ is a solution of the nonlinear singular twopoint boundary-value problem (1.5); furthermore, $u<v$ for $0<x<a, 0<t<T_{a}$.

(c) If $T_{a}=\infty$, and $\lim _{t \rightarrow \infty} u(M(t), t)=1^{-}$, then $T_{a+\varepsilon}<\infty$ for any $\varepsilon>0$.

Theorem 1(b) implies that there exists a critical length $a^{*}$ such that for $a<a^{*}, u$ exists for $0 \leq x \leq a, 0<t<\infty$. On the other hand, it follows from Theorem 1 (c) that $u$ quenches for $a>a^{*}$. The critical length $a^{*}$ is determined by the supremum of all values $a(>0)$ such that a solution of the problem (1.5) exists; if it exists for $a=a^{*}$, then $u$ also exists there.

The general solution (cf. Andrews [6], p. 229) of the singular equation,

$$
l w=0 \text {, }
$$

where

$$
l w=\left(x^{b} w^{\prime}\right)^{\prime}+c^{2} x^{b} w
$$

and $c$ is a positive constant, is given by

$$
w=x^{\nu}\left[c_{1} J_{\nu}(c x)+c_{2} Y_{\nu}(c x)\right]
$$

here, $\nu=(1-b) / 2, c_{1}$ and $c_{2}$ are arbitrary constants, and $J_{\nu}$ and $Y_{\nu}$ are Bessel functions of the first kind and the second kind, respectively. The singular SturmLiouville problem (2.1) and

$$
w(0)=0=w(a)
$$

has an eigenfunction $x^{\nu} J_{\nu}\left(j_{\nu} x / a\right)$ with the corresponding eigenvalue $\left(j_{\nu} / a\right)^{2}$, where $j_{\nu}$ denotes the first positive zero of $J_{\nu}$.

Let us consider the differential inequality,

$$
L \omega \geq-(1-\omega)^{-1}, \quad 0<x<a, \quad 0<t<T_{a},
$$

subject to

$$
\omega(x, 0)=0 \text { for } 0 \leq x \leq a, \quad \omega(0, t)=0=\omega(a, t) \text { for } 0<t<T_{a} .
$$

It follows from the maximum principle (cf. Protter and Weinberger ([24], pp. 168170) that if $u \leq A$ and $\omega \leq A$ for some positive constant $A<1$, then $u \geq \omega$.

Let us seek a lower bound $\omega$ of $u$ in the form $x^{\nu} J_{\nu}\left(j_{\nu} x / a\right) f(t)$. From (2.4),

$$
f^{\prime}(t)+\left(\frac{j_{\nu}}{a}\right)^{2} f(t) \leq\left\{x^{\nu} J_{\nu}\left(\frac{j_{\nu}}{a} x\right)\left[1-x^{\nu} J_{\nu}\left(\frac{j_{\nu}}{a} x\right) f(t)\right]\right\}^{-1} \text {. }
$$

Since

$$
\frac{d}{d z}\left[z^{\nu} J_{\nu}(z)\right]=z^{\nu} J_{\nu-1}(z)
$$

it follows that the derivative of the right-hand side of (2.5) with respect to $x$ is given by

$$
-\frac{j_{\nu} J_{\nu-1}\left(j_{\nu} x / a\right)\left[1-2 x^{\nu} J_{\nu}\left(j_{\nu} x / a\right) f(t)\right]}{a x^{\nu} J_{\nu}^{2}\left(j_{\nu} x / a\right)\left[1-x^{\nu} J_{\nu}\left(j_{\nu} x / a\right) f(t)\right]^{2}}
$$


Because the left-hand side of (2.5) does not depend on $x$, let us take the infimum of its right-hand side with respect to $x$. From (2.6), this infimum occurs at $x=a j_{\nu-1} / j_{\nu}$ for $f(t) \in\left(0,(2 m)^{-1}\right]$, where $m=\left(a j_{\nu-1} / j_{\nu}\right)^{\nu} J_{\nu}\left(j_{\nu-1}\right)$; since the infimum of the quantity $[y(1-y)]^{-1}$ is 4 , we have

$$
\frac{f^{\prime}(t)}{f(t)}+\left(\frac{j_{\nu}}{a}\right)^{2} \leq \begin{cases}\{m f(t)[1-m f(t)]\}^{-1} & \text { for } 0<f(t) \leq(2 m)^{-1} \\ 4 & \text { for } \quad(2 m)^{-1}<f(t) \leq m^{-1}\end{cases}
$$

Since $\omega(x, 0)=0$, it follows that $f(0)=0$.

We may determine $f(t)$ from (2.7) by using the equality sign. For $0<f(t) \leq$ $(2 m)^{-1}$, we have

$$
\frac{m(1-m f) d f}{1-\left(j_{\nu} / a\right)^{2} m f(1-m f)}=d t
$$

Let $t_{0}$ be the time when $f\left(t_{0}\right)=(2 m)^{-1}$. Upon integration from $t=0$ to $t=t_{0}$, we obtain

$t_{0}=\frac{a}{j_{\nu}}\left[4-\left(\frac{j_{\nu}}{a}\right)^{2}\right]^{-1 / 2} \tan ^{-1}\left\{\frac{j_{\nu}}{a}\left[4-\left(\frac{j_{\nu}}{a}\right)^{2}\right]^{-1 / 2}\right\}-\frac{1}{2}\left(\frac{a}{j_{\nu}}\right)^{2} \ln \left[1-\left(\frac{j_{\nu}}{2 a}\right)^{2}\right]$.

The solution of the problem,

is

$$
\frac{f^{\prime}(t)}{f(t)}+\left(\frac{j_{\nu}}{a}\right)^{2}=4, \quad f\left(t_{0}\right)=(2 m)^{-1},
$$

$$
f(t)=(2 m)^{-1} \exp \left\{\left[4-\left(j_{\nu} / a\right)^{2}\right]\left(t-t_{0}\right)\right\} .
$$

Since $\omega$ is a lower bound of $u$, the time $t_{1}$ such that $m f\left(t_{1}\right)=1$ is an upper bound of the quenching time $T_{a}$. Now,

$$
m^{-1}=(2 m)^{-1} \exp \left\{\left[4-\left(j_{\nu} / a\right)^{2}\right]\left(t_{1}-t_{0}\right)\right\}
$$

gives

$$
t_{1}=t_{0}+(\ln 2) /\left[4-\left(j_{\nu} / a\right)^{2}\right] \text {. }
$$

From the expression for $t_{0}$, we note that $4>\left(j_{\nu} / a\right)^{2}$, which implies that any length $a>j_{\nu} / 2$ leads to quenching. Thus, we obtain the following result.

THEOREM 2.

(a) The critical length, $a^{*} \leq j_{\nu} / 2$.

(b) At quenching, $T_{a} \leq t_{1}$.

We note, in particular, that Theorem 2 (a) generalizes the result of Walter since for $b=0$, we have $J_{\nu}(z)=[2 /(\pi z)]^{1 / 2} \sin z$, which gives $j_{\nu}=\pi$, and hence $a^{*} \leq \pi / 2$ with $a>\pi / 2$ implying quenching as given by Walter.

From Theorem 2(b), we obtain

$$
\lim _{a \rightarrow \infty} T_{a} \leq(3+2 \ln 2) / 8
$$

We also note that an upper bound $U(t)$, independent of $x$, is given by the initial value problem:

$$
U^{\prime}=\frac{1}{1-U}, \quad U(0)=0 .
$$

This gives $U(t)=1-(1-2 t)^{1 / 2}$. Therefore, there is no quenching for all values $a$ if $t<\frac{1}{2}$ (cf. Walter [27]). 
3. Global existence. To find $a^{*}$, we find the minimal solution of the nonlinear singular two-point boundary-value problem (1.5) for some $c a<j_{\nu}$. We construct a sequence, $\left\{v_{n}: n=1,2,3, \ldots\right\}$, by letting $v_{0} \equiv 0$, and for $n=0,1,2, \ldots$,

$$
l v_{n+1}=x^{b}\left[c^{2} v_{n}-\left(1-v_{n}\right)^{-1}\right], \quad v_{n+1}(0)=0=v_{n+1}(a) .
$$

Since $b<1$, it follows from (2.2) that the problem (2.1) and (2.3) for $c a<j_{\nu}$ has the trivial solution only. Thus for each value of $n$, the problem (3.1) has a unique solution. By using the properties of determinants and the fact that the Wronskian (cf. McLachlan [23], p. 197) of $J_{\nu}(z)$ and $Y_{\nu}(z)$ is $2 \pi / z$, Green's function $G(x ; \xi)$ corresponding to the operator $-l$ is given by

$$
G(x ; \xi)= \begin{cases}-\pi y_{1}(x) y_{2}(\xi) / 2, & 0 \leq x \leq \xi \\ -\pi y_{1}(\xi) y_{2}(x) / 2, & \xi \leq x \leq a\end{cases}
$$

where

$$
\begin{aligned}
y_{1}(x) & =x^{\nu} J_{\nu}(c x), \\
y_{2}(x) & =x^{\nu}\left[Y_{\nu}(c x)-k J_{\nu}(c x)\right], \\
k & =Y_{\nu}(c a) / J_{\nu}(c a) .
\end{aligned}
$$

Although the maximum principle (cf. Protter and Weinberger [24], p. 6) can be used to show that $G(x ; \xi)$ is positive, we give a more elementary proof here.

LEMMA 3. For $x$ and $\xi$ in the interval $(0, a)$ where $c a<j_{\nu}, G(x ; \xi)>0$.

Proof. It is sufficient to show that $y_{2}(x)<0$. It follows from the value of the Wronskian that

$$
\frac{d}{d x}\left[\frac{Y_{\nu}(c x)}{J_{\nu}(c x)}\right]=\frac{2}{\pi x J_{\nu}^{2}(c x)}>0 \quad \text { for } 0<x<a
$$

Hence,

$$
k>Y_{\nu}(c x) / J_{\nu}(c x)
$$

from which the lemma follows.

For $n=0,1,2, \ldots$,

$$
v_{n+1}(x)=\int_{0}^{a} \xi^{b} G(x ; \xi)\left\{\left[1-v_{n}(\xi)\right]^{-1}-c^{2} v_{n}(\xi)\right\} d \xi .
$$

Thus, the sequence is well defined.

For future reference, we state the following lemma, whose proof follows from the fact that $G(x ; \xi)$ is positive.

LEMMA 4. If $q(x)$ is continuous, nontrivial, and nonpositive, then the solution of the problem $l w=q$ subject to $(2.3)$ is positive for $0<x<a$.

The following theorem gives existence constructively.

THEOREM 5. If $0<c \leq 1$, then the nonlinear singular two-point boundary-value problem (1.5) has the minimal solution $v(<1)$, to which the sequence $\left\{v_{n}: n=\right.$ $0,1,2, \ldots\}$ converges strictly monotonically upwards.

Proof. From Lemma 4, $v_{1}>0$ for $0<x<a$. From (1.5),

$$
\frac{d^{2} v}{d x^{2}}+\frac{b}{x} \frac{d v}{d x}<0 \quad \text { for } \quad v<1, \quad v(0)=0=v(a) .
$$


It follows from the maximum principle that $v>0$ for $0<x<a$. Now,

$$
l\left(v-v_{1}\right)=-x^{b} v\left[1-c^{2}(1-v)\right] /(1-v)<0 .
$$

It follows from Lemma 4 that $v>v_{1}$ for $0<x<a$. Similarly, we have $v>v_{2}>v_{1}$.

Let us assume that for a particular value of $n$, say $j(\geq 2)$,

$$
0<v_{j-1}<v_{j}<v, \quad 0<x<a .
$$

An argument analogous to the above shows that

$$
0<v_{j-1}<v_{j}<v_{j+1}<v, \quad 0<x<a .
$$

By the principle of mathematical induction,

$$
0<v_{n}<v_{n+1}<v \quad \text { for } 0<x<a, \quad n=1,2,3, \ldots
$$

Since the strictly monotone sequence $\left\{v_{n}\right\}$ is bounded above and below, there exists a function $V(x)$ such that $\lim _{n \rightarrow \infty} v_{n}=V$. Let us denote the integrand of (3.3) by $f_{n}$. Because $\partial f_{n} / \partial v_{n}>0$, it follows from the Monotone Convergence Theorem (cf. Royden [25], p. 84) that

$$
V(x)=\int_{0}^{a} \xi^{b} G(x ; \xi)\left\{[1-V(\xi)]^{-1}-c^{2} V(\xi)\right\} d \xi .
$$

This implies that $V$ is a solution of the problem (1.5). Because of (3.4), $V$ is the minimal solution.

LEMMA 6. The rate of convergence of the iteration scheme (3.1) to the minimal solution of the nonlinear singular two-point boundary-value problem (1.5) is maximized by choosing $c=1$.

Proof. Let $c=1$ in (3.1). We obtain a sequence $\left\{V_{n}\right\}$ given by $V_{0} \equiv 0$, and for $n=0,1,2, \ldots$,

$$
\begin{gathered}
l V_{n+1}=x^{b}\left[V_{n}-\left(1-V_{n}\right)^{-1}\right], \\
V_{n+1}(0)=0=V_{n+1}(a) .
\end{gathered}
$$

Thus,

$$
\left[x^{b}\left(V_{1}-v_{1}\right)^{\prime}\right]^{\prime}+x^{b}\left(V_{1}-v_{1}\right)=-x^{b}\left(1-c^{2}\right) v_{1}<0 .
$$

By Lemma 4, $V_{1}>v_{1}$.

Let us assume that $V_{j}>v_{j}$. We have

$$
\left[x^{b}\left(V_{j+1}-v_{j+1}\right)^{\prime}\right]^{\prime}+x^{b}\left(V_{j+1}-v_{j+1}\right)<0 .
$$

By Lemma 4, $V_{j+1}>v_{j+1}$. Lemma 6 then follows from the principle of mathematical induction.

The next result shows that each successive approximation $v_{n}$ attains its maximum value at the same value of $x$.

THEOREM 7. If $v_{1}(x)$ attains its maximum value at $x=h$, then for $n=2,3,4, \ldots$, $v_{n}(x)$ also attains its maximum value at $x=h$.

Proof. From (3.1),

$$
v_{1}(x)=\int_{0}^{a} \xi^{b} G(x ; \xi) d \xi .
$$


Let us assume that for a particular value of $n$, say $j$, $v_{j}$ attains its maximum value at $x=h$. By the Weighted Mean Value Theorem for Integrals (cf. Burden and Faires [10], p. 4),

$$
v_{j+1}(x)=\left\{\left[1-v_{j}(\eta)\right]^{-1}-c^{2} v_{j}(\eta)\right\} \int_{0}^{a} \xi^{b} G(x ; \xi) d \xi,
$$

where $0<\eta<a$, and $\eta$ depends on $x$. By elementary calculus, the quantity inside the brace brackets attains its maximum at $x=h$. Thus, the maximum value of $v_{j+1}(x)$ is attained at $x=h$. The theorem then follows from the principle of mathematical induction.

Let $\Gamma$ denote the gamma function. The following lemma is useful in determining $h$ numerically.

LEMMA 8. The value $h$, at which $v_{1}$ attains its maximum, is the (unique) solution of the equation,

Proof. By using

$$
Y_{\nu-1}(c x)-\left[\frac{2^{\nu} \Gamma(\nu)}{\pi(a c)^{\nu} J_{\nu}(c a)}+k\right] J_{\nu-1}(c x)=0 .
$$

$$
\begin{gathered}
-\frac{d}{d z}\left[z^{1-\nu} J_{\nu-1}(z)\right]=z^{1-\nu} J_{\nu}(z), \\
-\frac{d}{d z}\left[z^{1-\nu} Y_{\nu-1}(z)\right]=z^{1-\nu} Y_{\nu}(z), \\
J_{\nu-1}(z) Y_{\nu}(z)-J_{\nu}(z) Y_{\nu-1}(z)=-2 /(\pi z),
\end{gathered}
$$

we obtain

$$
v_{1}(x)=\frac{1}{c^{2}}\left[\left(\frac{x}{a}\right)^{\nu} \frac{J_{\nu}(c x)}{J_{\nu}(c a)}-1\right]+\frac{\pi c^{\nu-2} x^{\nu}}{2^{\nu} \Gamma(\nu)}\left[k J_{\nu}(c x)-Y_{\nu}(c x)\right]
$$

By using

we get

$$
\begin{aligned}
\frac{d}{d z}\left[z^{\nu} J_{\nu}(z)\right] & =z^{\nu} J_{\nu-1}(z), \\
\frac{d}{d z}\left[z^{\nu} Y_{\nu}(z)\right] & =z^{\nu} Y_{\nu-1}(z),
\end{aligned}
$$

$$
v_{1}^{\prime}(x)=\frac{x^{\nu} J_{\nu-1}(c x)}{c a^{\nu} J_{\nu}(c a)}+\frac{\pi c^{\nu-1} x^{\nu}}{2^{\nu} \Gamma(\nu)}\left[k J_{\nu-1}(c x)-Y_{\nu-1}(c x)\right] .
$$

For critical values $H, v_{1}^{\prime}(x)=0$, from which (3.5) follows.

Using $v_{1}^{\prime}(H)=0$, and the formulae,

$$
\begin{aligned}
& J_{\nu}^{\prime}(z)=\nu J_{\nu}(z) / z-J_{\nu+1}(z), \\
& Y_{\nu}^{\prime}(z)=\nu Y_{\nu}(z) / z-Y_{\nu+1}(z),
\end{aligned}
$$

we obtain

$$
v_{1}^{\prime \prime}(H)=-H^{\nu}\left\{\frac{J_{\nu}(c H)}{a^{\nu} J_{\nu}(c a)}+\frac{\pi c^{\nu}}{2^{\nu} \Gamma(\nu)}\left[k J_{\nu}(c H)-Y_{\nu}(c H)\right]\right\} .
$$

Since $\nu>0$, it follows from $(3.2)$ that $v_{1}^{\prime \prime}(H)<0$, which shows that all critical points are relative maxima. Hence, there can only be one maximum, and the lemma is proved.

The following result shows that if once the difference between two successive iterates gets bigger, then the sequence $\left\{v_{n}\right\}$ constructed by (3.1) diverges. 
LEMMA 9. For $0<x<a$, if $v_{n+1}-v_{n}>v_{n}-v_{n-1}$ for some positive integer $n$, then $v_{m+1}-v_{m}>v_{m}-v_{m-1}$ for $m=n+1, n+2, n+3, \ldots$.

Proof. Since the sequence $\left\{v_{n}\right\}$ is strictly increasing, it follows that

$$
\begin{aligned}
v_{n+2}-v_{n+1} & =\int_{0}^{a} \xi^{b} G(x ; \xi)\left(v_{n+1}-v_{n}\right)\left\{\left[\left(1-v_{n+1}\right)\left(1-v_{n}\right)\right]^{-1}-c^{2}\right\} d \xi \\
& >\int_{0}^{a} \xi^{b} G(x ; \xi)\left(v_{n}-v_{n-1}\right)\left\{\left[\left(1-v_{n}\right)\left(1-v_{n-1}\right)\right]^{-1}-c^{2}\right\} d \xi \\
& =v_{n+1}-v_{n} .
\end{aligned}
$$

The lemma then follows by using mathematical induction.

The following result shows that the maximum difference between two successive approximations occurs at $h$.

LEMma 10. For $0<c \leq 1$, the difference between two successive iterates constructed by (3.1) attains its maximum at $x=h$.

Proof. Since $v_{n+1}>v_{n}$ for $0<x<a$, it follows from (3.1) that for $n=0,1,2, \ldots$,

$$
l\left(v_{n+1}-v_{n}\right)<0 .
$$

At $x=h,\left(v_{n+1}-v_{n}\right)^{\prime}=0$. From (3.8), $\left(v_{n+1}-v_{n}\right)^{\prime \prime}(h)<0$. The lemma then follows.

We remark that Lemmas 9 and 10 can be used to conclude for $0<c \leq 1$ that once the difference between two successive approximations at $h$ gets bigger, then we have quenching for that choice of $a$. On the other hand, Lemma 10 may also be used to stop the computation of the minimal solution $v$ if the difference between two successive iterates at $h$ is smaller than the accuracy desired. Both these lemmas are useful in saving computer time.

4. Numerical results. We apply our results in Secs. 2 and 3 to compute critical lengths numerically with the use of a computer for various given values of $b$.

For any given $b$, we obtain $\nu=(1-b) / 2$. This in turn determines the first positive zero $j_{\nu}$ of $J_{\nu}$ (cf. Jahnke and Emde [16], p. 167). By Theorem 2(a), $a^{*} \leq j_{\nu} / 2$. We can take this value $j_{\nu} / 2$ as the starting point for finding $a^{*}$. Let us take $a=j_{\nu} / 2-\delta$ (> 0 ), where $\delta$ is some small positive constant (say, 0.1$)$. We compute $v_{n+1}(x)$ by using the representation formula (3.3) with $v_{0} \equiv 0$, and $c=1$ for maximum rate of convergence. To compute $v_{n+1}$, we use subroutines from the IMSL Library (Edition 9.2; Revised November, 1984; IMSL LIB-0009).

1. When $\nu$ is not a nonnegative integer, we evaluate $k=Y_{\nu}(a) / J_{\nu}(a)$ by using subroutines MMBSYN (to compute, to double precision, Bessel functions of the second kind of nonnegative real fractional order for real positive arguments), and MMBSJR (to compute, to double precision, Bessel functions of the first kind of nonnegative real order for real positive arguments); when $\nu$ is a nonnegative integer, we use MMBSJN instead of MMBSJR.

2. By using (3.6), (3.7), and the subroutine MGAMAD (= DGAMMA to evaluate the gamma function of a double precision argument), Newton's method is used to find the zero $h$ from (3.5).

3. We divide the interval $[0, a]$ into 20 equal subintervals, and replace the subdivision point closest to $h$ by $h$. To save computer time, we compute and store all possible 
values of $\xi^{1-\nu} J_{\nu}(\xi)$ and $\xi^{1-\nu}\left[Y_{\nu}(\xi)-k J_{\nu}(\xi)\right]$, where $\xi$ denotes the subdivision point according to the algorithm of Romberg integration.

4. At the nineteen interior subdivision points chosen in step 3 , we evaluate $x^{\nu} J_{\nu}(x)$ and $x^{\nu}\left[Y_{\nu}(x)-k J_{\nu}(x)\right]$, and store them in the memory of the computer.

5. The subroutine ICSCCU (cubic spline interpolation in single precision) is used to interpolate the functions $v_{n}$ at the 21 subdivision points: $0=x_{1}<x_{2}<x_{3}<\cdots<$ $x_{21}=a$.

6. Let

$$
\begin{aligned}
& F_{n, 1}\left(x_{i}, x_{j}\right)=\frac{\pi}{2} \int_{x_{i}}^{x_{j}} \xi^{b} y_{1}(\xi)\left\{c^{2} v_{n}(\xi)-\left[1-v_{n}(\xi)\right]^{-1}\right\} d \xi \\
& F_{n, 2}\left(x_{i}, x_{j}\right)=\frac{\pi}{2} \int_{x_{i}}^{x_{j}} \xi^{b} y_{2}(\xi)\left\{c^{2} v_{n}(\xi)-\left[1-v_{n}(\xi)\right]^{-1}\right\} d \xi .
\end{aligned}
$$

To save computer time in obtaining $v_{n+1}$ from $v_{n}$, we evaluate $v_{n+1}\left(x_{11}\right)$ first. From (3.3),

$$
v_{n+1}\left(x_{11}\right)=y_{2}\left(x_{11}\right) F_{n, 1}\left(x_{1}, x_{11}\right)+y_{1}\left(x_{11}\right) F_{n, 2}\left(x_{11}, x_{21}\right) \text {. }
$$

To obtain $v_{n+1}\left(x_{10}\right)$, we only need to compute $F_{n, 1}\left(x_{10}, x_{11}\right)$ and $F_{n, 2}\left(x_{10}, x_{11}\right)$ since

$$
\begin{aligned}
v_{n+1}\left(x_{10}\right)=y_{2}\left(x_{10}\right)\left[F_{n, 1}\left(x_{1}, x_{11}\right)-F_{n, 1}\right. & \left.\left(x_{10}, x_{11}\right)\right] \\
& +y_{1}\left(x_{10}\right)\left[F_{n, 2}\left(x_{11}, x_{21}\right)+F_{n, 2}\left(x_{10}, x_{11}\right)\right] .
\end{aligned}
$$

In this way, we can successively compute $v_{n+1}$ at $x_{10}, x_{9}, x_{8}, \ldots, x_{2}$. Similarly,

$$
\begin{aligned}
v_{n+1}\left(x_{12}\right)=y_{2}\left(x_{12}\right)\left[F_{n, 1}\left(x_{1}, x_{11}\right)+F_{n, 1}\right. & \left.\left(x_{11}, x_{12}\right)\right] \\
& +y_{1}\left(x_{12}\right)\left[F_{n, 2}\left(x_{11}, x_{21}\right)-F_{n, 2}\left(x_{11}, x_{12}\right)\right] .
\end{aligned}
$$

Proceeding in this way, we obtain successively $v_{n+1}$ at $x_{12}, x_{13}, x_{14}, \ldots, x_{20}$. The subroutine DCADRE (to perform, to single precision, numerical integration of a function using cautious adaptive Romberg extrapolation) is used to perform the integration. To do so, we use the subroutine ICSEVU (for evaluation of a cubic spline to single precision) to evaluate $v_{n}$ in the integrand. We note that most of the values $\xi^{1-\nu}\left[Y_{\nu}(\xi)-k J_{\nu}(\xi)\right]$, and $\xi^{1-\nu} J_{\nu}(\xi)$ are already stored in the memory of the computer (by step 3); if a particular one is not there, then it is computed. In this way, we obtain $v_{1}, v_{2}, v_{3}, \ldots$

7. We stop the computation of $v_{n+1}$ in three ways.

(a) If $v_{n}(h) \geq 1$, then $u$ quenches.

(b) If at $h, v_{n+1}-v_{n}>v_{n}-v_{n-1}$ for some $n$, then $u$ quenches.

(c) If $v_{n}(h)<1$, and $v_{n+1}(h)-v_{n}(h)<5 \times 10^{-(r+1)}$ (for an accuracy to $r$ decimal points), then $u$ exists globally.

If $u$ quenches, then we use $a=j_{\nu} / 2-2 \delta(>0)$. In this way, we reach $a=j_{\nu} / 2-p \delta$ for some positive integer $p$ when $u$ exists globally. We then use the method of bisection to determine the value $a^{* *}$ between $j_{\nu} / 2-(p-1) \delta$ and $j_{\nu} / 2-p \delta$ such that for $a \leq a^{* *}$, we have global existence, and for $a>a^{* *}$, we have quenching. Now, $a^{*}-a^{* *}<5 \times 10^{-(r+1)}$. Since $a^{*}-a^{* *}$ can be made as small as we like, $a^{* *}$ can be taken numerically to be $a^{*}$. 
The results for four values of $b$ with $r=4$ are given in Table 1 .

TABLE 1. Critical lengths corresponding to different values of $b$, and upper bounds for quenching time when $a=\pi$.

\begin{tabular}{c|c|c|c|c}
$b$ & $\nu$ & $j_{\nu} / 2$ & $a^{*}$ & $t_{1}$ \\
\hline 0.4 & 0.3 & 1.4271 & 1.3935 & 0.6496 \\
0.0 & 0.5 & 1.5708 & 1.5303 & 0.6772 \\
-0.4 & 0.7 & 1.7110 & 1.6544 & 0.7099 \\
-1.0 & 1.0 & 1.9159 & 1.8239 & 0.7715
\end{tabular}

Two graphs are given below to illustrate the other results before quenching occurs. Figure 1 shows how much the maximum of $v$ shifts away from the center as $b$ is changed by plotting the position $h$ (where the maximum occurs) against $b$ for a fixed given value of $a$. Figure 2 shows the height $v(h)$ of the maximum as a function of $a$ for three different values of $b$.

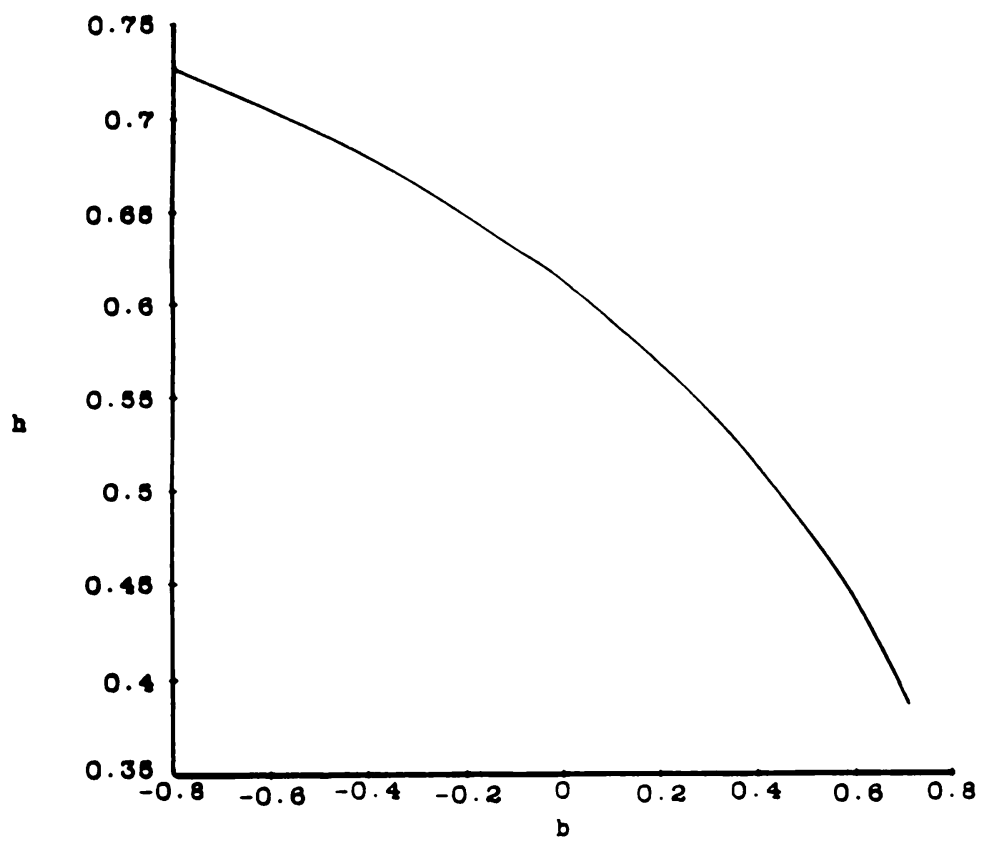

FIG. 1. Position $h$ versus $b$ for $a=1.2300$. 


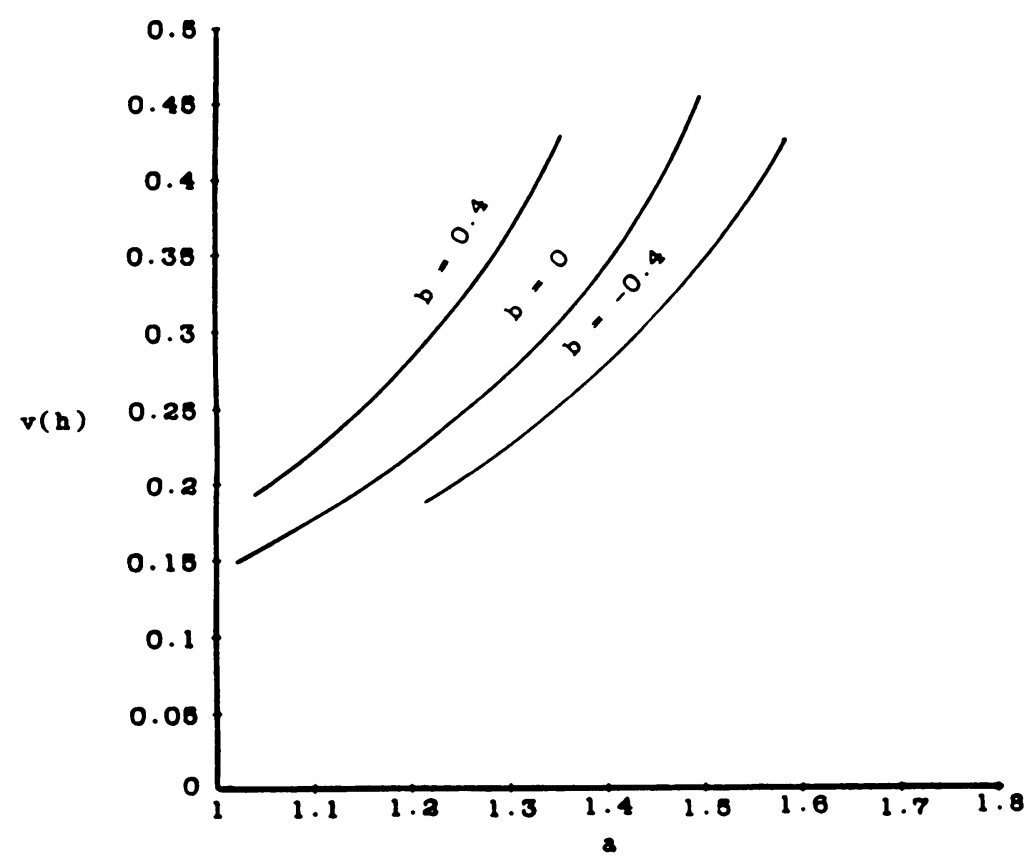

FIG. 2. Height $v(h)$ versus $a$.

\section{REFERENCES}

[1] A. Acker and W. Walter, The quenching problem for nonlinear parabolic differential equations, Lecture Notes in Math. 564, Springer-Verlag, New York, 1976, pp. 1-12

[2] A. Acker and W. Walter, On the global existence of solutions of parabolic differential equations with a singular nonlinear term, Nonlinear Anal. 2, 499-505 (1978)

[3] V. Alexiades, $A$ singular parabolic initial-boundary value problem in a noncylindrical domain, SIAM J. Math. Anal. 11, 348-357 (1980)

[4] V. Alexiades, Generalized axially symmetric heat potentials and singular parabolic initial-boundary value problems, Arch. Rat. Mech. Anal. 79, 325-350 (1982)

[5] V. Alexiades and C. Y. Chan, $A$ singular Fourier problem with nonlinear radiation in a noncylindrical domain, Nonlinear Anal. 5, 835-844 (1981)

[6] L. C. Andrews, Special functions for engineers and applied mathematicians, Macmillan Publishing Co., New York, 1985, p. 229

[7] O. Arena, On a singular parabolic equation related to axially symmetric heat potentials, Ann. Mat. Pura Appl. (4) 105, 347-393 (1975)

[8] L. Bragg, The radial heat polynomials and related functions, Trans. Amer. Math. Soc. 119, 270-290 (1965)

[9] H. Brezis, W. Rosenkrantz, and B. Singer, with an appendix by P. Lax, On a degenerate ellipticparabolic equation occurring in the theory of probability, Comm. Pure Appl. Math. 24, 395-416 (1971)

[10] R. L. Burden and J. D. Faires, Numerical analysis, 3rd ed., Prindle, Weber and Schmidt, Boston, 1985, p. 4

[11] C. Y. Chan and Y. C. Hon, A constructive solution for a generalized Thomas-Fermi theory of ionized atoms, Quart. Appl. Math. 45, 591-599 (1987)

[12] P. H. Chang and H. A. Levine, The quenching of solutions of semilinear hyperbolic equations, SIAM J. Math. Anal. 12, 893-903 (1981)

[13] F. Cholewinski and D. Haimo, The Weierstrass-Hankel convolution transform, J. Analyse Math. 17, $1-58(1966)$ 
[14] D. Colton, Cauchy's problem for a singular parabolic differential equation, J. Differential Equations 8, 250-257 (1970)

[15] D. Haimo, Series representation of generalized temperature functions, SIAM J. Appl. Math. 15, 359367 (1967)

[16] E. Jahnke and F. Emde, Table of functions with formulae and curves, 4th ed., Dover Publications, New York, 1945, p. 167

[17] H. Kawarada, On solutions of initial-boundary problem for $u_{t}=u_{x x}+1 /(1-u)$, Publ. Res. Inst. Math. Sci. 10, Kyoto Univ., 729-736 (1975)

[18] J. Lamperti, A new class of probability theorems, J. Math. Mech. 11, 749-772 (1962)

[19] H. A. Levine, The quenching of solutions of linear parabolic and hyperbolic equations with nonlinear boundary conditions, SIAM J. Math. Anal. 14, 1139-1153 (1983)

[20] H. A. Levine, The phenomenon of quenching. a survey, in V. Lakshmikantham (ed.), Trends in the Theory and Practice of Non-linear Analysis, North Holland, New York, 1985, pp. 275-286

[21] H. A. Levine and G. M. Lieberman, Quenching of solutions of parabolic equations with nonlinear boundary conditions in several dimensions, J. Reine Angew. Math. 345, 23-38 (1983)

[22] H. A. Levine and J. T. Montgomery, The quenching of solutions of some nonlinear parabolic equations, SIAM J. Math. Anal. 11, 842-847 (1980)

[23] N. W. McLachlan, Bessel functions for engineers, 2nd ed., Oxford University Press, London, 1955, p. 197

[24] M. H. Protter and H. F. Weinberger, Maximum principles in differential equations, Prentice Hall, Englewood Cliffs, 1967, pp. 6 and 168-170

[25] H. L. Royden, Real analysis, 2nd ed., Macmillan Publishing Co., New York, 1968, p. 84

[26] A. D. Solomon, Melt time and heat flux for a simple PCM body, Solar Energy 22, 251-257 (1979)

[27] W. Walter, Parabolic differential equations with a singular nonlinear term, Funkcial. Ekvac. 19, 271277 (1976) 